\title{
THE MINIMAL COOLING OF NEUTRON STARS *
}

\author{
DANY PAGE \\ Instituto de Astronomía, UNAM \\ Mexico, D.F. 04340, MEXICO \\ E-mail:page@astroscu.unam.mx
}

\begin{abstract}
A general overview of the main physical processes driving the cooling of an isolated neutron star is presented. Among the most important ones are the various possible neutrino emission processes and the occurrence of baryon pairing. Special emphasis is also put on the importance of the chemical composition of the upper layers of the star. A detailed analysis of a Minimal Scenario, which explicitly postulates that no "exotic" form of matter be present, is summarized and compared with presently available observational data. No striking incompatibility of the data with the predictions of the Minimal Scenario is found. Nevertheless, two, possibly three, conspicuous stars are identified which may, when better data are available, constitute strong astrophysical evidence for the occurrence of a new state of matter at high density.
\end{abstract}

\section{Introduction}

Among the various ways to search for new states of matter at high density the study of neutron stars is a promising one. Many aspects of the very diverse phenomenology of these stars can provide us with indications of such "exotic" matter (see, e.g., Ref. [2]). In particular, the modeling of the thermal evolution of isolated neutron stars is an avenue along which much effort has been invested. Being born in a supernova at temperatures in excess of $3 \times 10^{11} \mathrm{~K}$, young neutron stars rapidly cool through neutrino emission and the cooling rate is a very sensitive function of the composition of matter at the most extreme densities present in their inner core. Different models predict central densities from around $4 \times \rho_{\text {nucl }}$ up to 15 to 20 times $\rho_{\text {nucl }}$ ( $\rho_{\text {nucl }}$ being the nuclear density), which may very probably be within the necessary range to see deconfinement of baryonic matter into quark matter. Less extreme models predict the occurrence of charged meson condensates

*Work in collaboration with J.M. Lattimer, M. Prakash, \& A. W. Steiner: Ref. [1] 
and/or hyperons populations. However, distinguishing between these various scenarios is a very delicate problem [3]. Finally, the most extreme model consider that neutron stars may convert into "Strange Stars" made entirely of deconfined quark matter which would have a completely different thermal evolution [4].

Evidence in favor of the presence of a new state of matter in the core of some neutron stars can only be obtained by finding some observed characteristics of these stars which cannot be understood without the assumption of the presence of such matter. Within this point of view I describe a "Minimal Scenario" of neutron star cooling, proposed recently in Ref. [1], which precisely assumes that the neutron star interior is devoid of any form of matter beyond the standard composition consisting of only neutrons with a small admixture of protons, accompanied by the necessary amount of electrons and muons to keep the star charge neutral. This Minimal Scenario is a revised modern version of the "Standard Scenario" but incorporates, as an essential ingredient, the effects of nucleon pairing, i.e., neutron superfluidity and/or proton superconductivity, on the star's specific heat and neutrino emission, particularly the neutrino emission by the very formation, and breaking, of the Cooper pairs. Comparison of the predictions of this Minimal Scenario with data may hence provide us with the long searched for evidence for "exotic" matter.

Section 2 briefly summarizes the presently available data on temperature and luminosity of isolated cooling neutron stars. Section 3 describes the most important input physics for the study of the Minimal Scenario. Section 4 compares the results with data and Section 5 offers conclusions.

An extensive presentation of this work can be found in Ref. [1] to which the present summary could be considered as a, hopefully convenient, Traveler's Guide.

\section{Observational Data}

Numerical calculations of neutron star cooling give as a natural result the evolution of the star's photon thermal luminosity $L$ as a function of time. This luminosity can equally well be described in terms of an effective temperature $T_{e}$ through the standard relation

$$
L \equiv 4 \pi R^{2} \cdot \sigma_{S B} T_{e}^{4} \quad \text { or } \quad L_{\infty} \equiv 4 \pi R_{\infty}^{2} \cdot \sigma_{S B} T_{e \infty}^{4}
$$

( $\sigma_{S B}$ being the Stefan-Boltzmann constant) where $R$ is the star's radius and the subscripts $\infty$ indicate quantities as observed "at infinity". 
Observations of cooling neutron stars can provide us with data in the form of luminosity $L_{\infty}$ and/or temperature $T_{\infty}$ at infinity. The measured $T_{\infty}$ depends of course on the kind atmosphere assumed in the spectral fits, realistic neutron star atmosphere models giving generally lower values than blackbodies. The measured $L_{\infty}$ is obtained from the total observed thermal flux, corrected for interstellar absorption, and the distance $D$. If $D$ is known with sufficient accuracy Eq. 1 could be used to determine $R_{\infty}$ [5] assuming that $T_{e}$ is also accurately known, i.e., that the correct atmosphere model has been used in the spectral fit. If the deduced $R_{\infty}$ is too small or too large compared to the "canonical" $10 \mathrm{~km}$ expected for a neutron star it is a strong indication that the atmosphere model is not correct. Nevertheless, some exotic models of compact stars as "Strange Stars" may result in small radii and also some magnetic field configurations may be able to confine the detectable surface thermal emission to an area significantly smaller than the whole surface of the star [6].

The data I will use are shown in Fig. 1 and have been selected according to this self-consistency $R_{\infty}$-criterium. Two types of spectra have been preferentially used in the spectral fits producing these data: blackbodies and magnetized hydrogen atmospheres. Only the second one has been successful in deducing acceptable values for $R_{\infty}$ and this lead to the selection of $T_{\infty}$ and $L_{\infty}$ of the objects plotted with thick lines in Fig. 1. For the objects plotted with thin lines the magnetized hydrogen atmosphere models require much too large radii while blackbodies seem more reasonable but usually on the low side of the expected range of $R_{\infty}$. Given this situation, for these
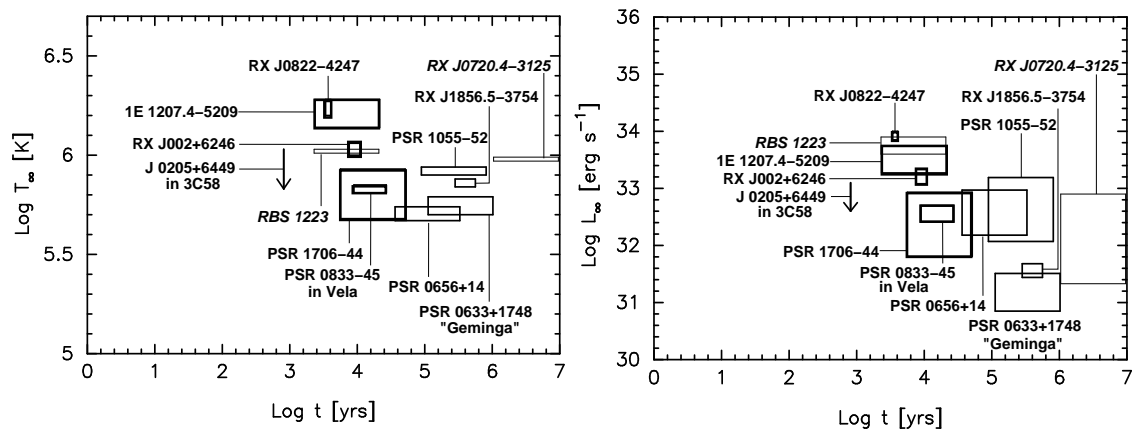

Figure 1. Measured $T_{\infty}$ and $L_{\infty}$, for twelve isolated neutron stars, versus age. The age, and their error bars, are either from kinematical information when available or from the pulsar spin-down time scale, in which case an uncertainty of a factor three has been assumed. See [1] for references and more details. 


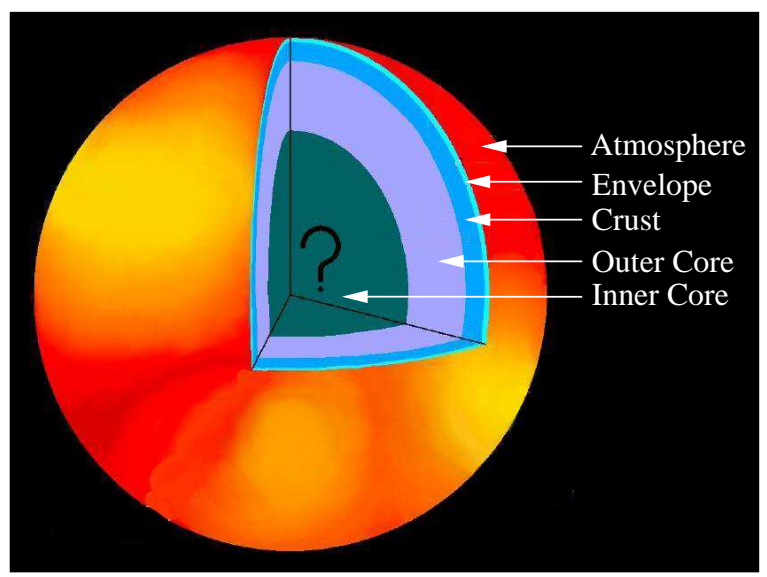

Figure 2. The different layers in a neutron star. [Drawing by the author.]

objects it is difficult to decide which of $T_{\infty}$ or $L_{\infty}$ is the more reliable value to use for comparison with the theoretical models and I hence prefer to use both, leaving the reader draw her/his own conclusions from the analysis.

\section{The Physics of Neutron Star Cooling}

The picture in Fig. 2 illustrates the most important layers in a neutron star:

- Atmosphere: at most a few tens of centimeters thick, this is the visible surface of the star (it may actually be a solid surface instead of an atmosphere) where the thermal photons are emitted. It is of utmost importance for observations since it is where the energy distribution of the thermally emitted photons, i.e., the thermal spectrum, is determined. However, since all the heat flowing into it from the interior is reemitted at the surface, the atmosphere does not affect the thermal evolution of the star.

- Envelope: this layer is several tens of meters thick and is, by definition, where a large temperature gradient is always present. It is a throttle which controls how much heat can leak out of the star and thus determines the relationship between the interior temperature and the effective temperature or, equivalently, the surface photon luminosity $L_{\gamma}$.

- Crust: with a thickness of several hundred meters, this layer is important mostly in the cooling of very young stars or in the study of transient phenomena as glitches. For our present purpose its only relevance is its (small) contribution to the specific heat. 
In both the envelope and the crust matter is made of nuclei immersed in a gas of electrons and, in the inner part of the crust, at densities higher than $\rho_{\text {drip }} \simeq 4.3 \times 10^{11} \mathrm{gm} \mathrm{cm}^{-3}$, a quantum liquid of dripped neutrons.

- Outer Core: region at densities higher than $\rho_{\mathrm{cc}} \simeq 1.6 \times 10^{14} \mathrm{gm} \mathrm{cm}^{-3}$, where matter is a quantum liquid predominantly composed of neutrons with a small fraction of protons, plus electrons and muons to maintain charge neutrality.

- Inner Core: the mysterious part, which may or may not exist, and where "exotic" forms of matter may appear. In the Minimal Scenario this inner core is explicitly assumed to be non-existent.

All calculations I will present here were performed with a wholly general relativistic Henyey-type stellar evolution code which solves exactly the equations of energy balance and heat transport inside a star whose structure is calculated by solving the Tolman-Oppenheimer-Volkov equation of hydrostatic equilibrium. Nevertheless, the most important features can be understood from the (Newtonian) energy conservation equation

$$
\frac{d E_{t h}}{d t}=C_{v} \frac{d T}{d t}=-L_{\nu}-L_{\gamma}+H
$$

where $E_{t h}$ is the thermal energy content of the star, $L_{\nu}$ the neutrino luminosity, $L_{\gamma}$ the surface photon luminosity and $H$ would give the contribution from "heating processes" as, e.g., friction within the differentially rotating neutron superfluid or magnetic field decay, and $C_{v}$ is the total specific heat, $T$ being the interior temperature. Solving the heat transport equation gives us the detailed temperature profile in the interior but within a few tens of years after its birth the star becomes isothermal and its evolution is then entirely controlled by (the GR version of) Eq. 2. At this time a significant temperature gradient is only present in the envelope (see, however, Ref. [6]).

\subsection{The envelope and the photon luminosity}

Once the star is isothermal its interior temperature is equal to the temperature at the bottom of the envelope, $T_{b}$, and the relationship between $T_{b}$ and the "surface", or effective, temperature is called the " $T_{b}-T_{e}$ relationship", which then gives us $L_{\gamma}$ through Eq. 1. A useful approximation to it is [7]

$$
T_{e} \sim \sqrt{T_{b}} \quad \text { with } \quad T_{e} \approx 10^{6} \mathrm{~K} \longleftrightarrow T_{b} \approx 10^{8} \mathrm{~K} .
$$

which gives, very roughly, $L_{\gamma} \sim T^{2}$. Nevertheless, significant deviation from Eq. 3 can occur. This equation is based on models which assumed that no magnetic field is present and that the envelope is made of iron, and 
iron-like, nuclei, but in case light elements, e.g., $\mathrm{H}, \mathrm{He}, \mathrm{C}$, or $\mathrm{O}$, are present they strongly reduce the blanketing effect of the envelope. A magnetic field also increases the heat permeability of the envelope in the regions where it is pointing radially but strongly suppresses it in regions where it makes small angles with the surface, thus inducing a highly non-uniform surface temperature distribution (see, e.g., Ref. [10]), and motivating the shaded surface in Fig. 2. Nevertheless the overall effect of the magnetic field is not as strong as the effect of the chemical composition. Figure 3 shows this $T_{b}-$ $T_{e}$ relationship for various models of envelope with varying amounts of light elements and an envelope formed entirely of heavy iron-like elements with and without a magnetic field. Notice that an envelope with a significant amount of light elements results, for a given interior temperature $T_{b}$, in a luminosity $L_{\gamma}$ which is more than one order of magnitude higher than an envelope made of heavy elements.

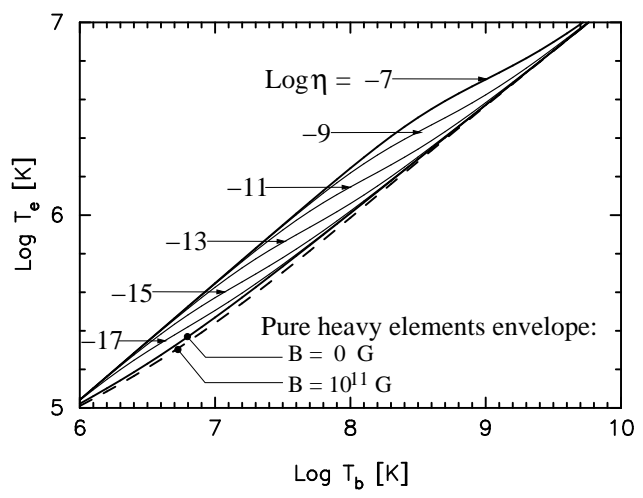

Figure 3. Relationship between the effective temperature $T_{e}$ and the interior temperature $T_{b}$ at the bottom of the envelope assuming various amounts of light elements parameterized by $\eta \equiv g_{s 14}^{2} \Delta M_{\mathrm{L}} / M$ where $\Delta M_{\mathrm{L}}$ is the mass in light elements in the envelope and $g_{s} 14$ the surface gravity in units of $10^{14} \mathrm{~cm} \mathrm{~s}^{-1}, M$ being the total star's mass, in the absence of a magnetic field [8]. Also shown are the $T_{b}-T_{e}$ relationships for an envelope of heavy elements with and without the presence of a dipolar field of strength of $10^{11} \mathrm{G}$ following Ref. [9].

The chemical composition of this envelope is probably determined by poorly understood processes occurring during the first hours of the life of the star, including post-supernova fall-back and also possible later accretion, bombardment by high energy $\gamma$-rays from the magnetosphere, ejection of light nuclei by the pulsar mechanism,... It is hence possibly totally unrelated to the interior structure of the star and may vary from star to star 
and/or evolve with time. We have no choice but consider it as a free parameter which has to be varied independently of the internal structure of the star, i.e., within both the Minimal Scenario and any other exotic one. Spectral fits to the thermal spectrum could determine the composition of the atmosphere: an iron atmosphere necessarily implies an heavy element envelope but a light element atmosphere unfortunately does not impose any restriction on the the chemical composition of the layers a few tens of centimeters beneath it.

\subsection{The neutrino luminosity}

The second important term in Eq. 2 is $L_{\nu}$ which is strongly dominated by the neutrino emission from the core. All significant processes are directly related to $\beta$ - and inverse $\beta$-decay of neutrons with protons and several of them are listed in Table 1 with their emissivities $q_{\nu}$. The simplest such process is the direct Urca ("DUrca") process. However, momentum conservation in this process requires proton fractions $x_{p}$ above $15 \%$ [11] while at nuclear density it is only of the order of $5 \%$. Thus, in the outer core of the neutron star, and this is the definition of the outer core, neutrino emission is due to the modified Urca (MUrca) process in which a second "spectator" nucleon (a neutron for the neutron branch or a proton in the proton branch of MUrca) contributes by giving or absorbing the extra momentum needed. Being a five fermion process instead of a three fermion one, the MUrca process is much less efficient than the DUrca process. It acquires two extra Pauli blocking actors $\left(T / E_{F}\right), E_{F}$ being the Fermi energy of the extra nucleon: since $E_{F} \sim 100 \mathrm{MeV}$, with $T=10^{9} \cdot T_{9} \mathrm{~K}$, the reduction of MUrca is of the order of $10^{-6} T_{9}^{2}$ compared to DUrca. Another possibility which allows the DUrca process, but with a reduced efficiency, is the presence of a charged meson $\left(\pi^{-}\right.$or $\left.K^{-}\right)$condensate which can easily contributes to momentum conservation without introducing any dramatic phase space limitation as a nucleon does in the MUrca process. In case hyperons, or quarks, appear at high density they will also participate in DUrca processes and enormously increase $L_{\nu}$.

In short, for the chemical composition expected at densities not too much higher than $\rho_{\text {nucl }}$ where the proton fraction is small the neutrino emission is due to the MUrca process while any change beyond this will increase the emissivity by many orders of magnitude. This MUrca process is the essence of the Standard Scenario for neutron star cooling but the occurrence of nucleon pairing and its proper treatment makes the subject more complicated and leads to the Minimal Scenario. I refer the reader to 
the two excellent reviews by Pethick [12] and Yakovlev et al. [13] for more details on neutrino emission processes.

Table 1. Some core neutrino emission processes and their emissivities.

\begin{tabular}{lcc}
\hline Process Name & Process & $\begin{array}{c}\text { Emissivity } q_{\nu} \\
\left(\mathrm{erg} / \mathrm{sec} / \mathrm{cm}^{3}\right)\end{array}$ \\
\hline a) Modified Urca & $\left\{\begin{array}{l}n^{\prime}+n \rightarrow n^{\prime}+p+e^{-}+\bar{\nu}_{e} \\
n^{\prime}+p+e^{-} \rightarrow n^{\prime}+n+\nu_{e}\end{array}\right.$ & $\sim 10^{21} \cdot T_{9}^{8}$ \\
b) K-condensate & $\left\{\begin{array}{l}n+K^{-} \rightarrow n+e^{-}+\bar{\nu}_{e} \\
n+e^{-} \rightarrow n+K^{-}+\nu_{e}\end{array}\right.$ & $\sim 10^{24} \cdot T_{9}^{6}$ \\
c) $\pi$ - condensate & $\left\{\begin{array}{l}n+\pi^{-} \rightarrow n+e^{-}+\bar{\nu}_{e} \\
n+e^{-} \rightarrow n+\pi^{-}+\nu_{e}\end{array}\right.$ & $\sim 10^{26} \cdot T_{9}^{6}$ \\
d) Direct Urca & $\left\{\begin{array}{l}n \rightarrow p+e^{-}+\bar{\nu}_{e} \\
p+e^{-} \rightarrow n+\nu_{e}\end{array}\right.$ & $\sim 10^{27} \cdot T_{9}^{6}$ \\
e) Quark Urca & $\left\{\begin{array}{l}d \rightarrow u+e^{-}+\bar{\nu}_{e} \\
u+e^{-} \rightarrow d+\nu_{e}\end{array}\right.$ & $\sim 10^{26} \alpha_{c} T_{9}^{6}$ \\
\hline
\end{tabular}

\subsection{Baryon paring}

Pairing of baryons, either nucleons or hyperons, and also of quarks if present, is predicted to occur in most of the interior of a neutron star. At low Fermi momenta neutrons and protons are expected to pair in a ${ }^{1} S_{0}$ angular momentum state while at higher momenta a ${ }^{3} P_{2}$ state is probably replacing it. The ${ }^{1} S_{0}$ neutron gap has been extensively studied and
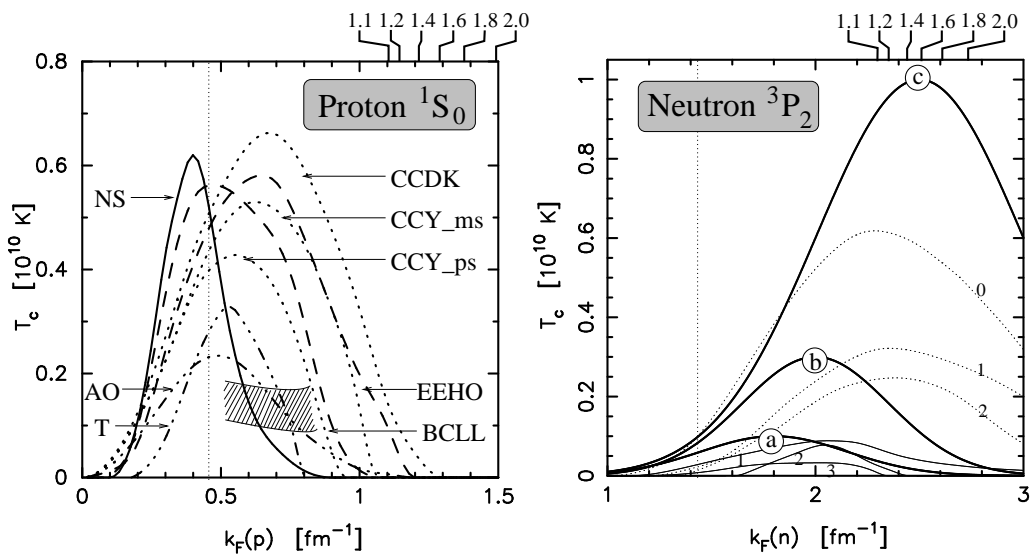

Figure 4. Predictions of critical temperatures $T_{c}$ for pairing of protons in the ${ }^{1} S_{0}$ state and neutrons in the ${ }^{3} P_{2}$ state. The dotted vertical lines indicate the crust-core boundary. Values of $k_{F}$ at center of stars of masses 1.1, 1.2, 1.4, 1.6, 1.8, and $2.0 M_{\odot}$ are marked at the upper margin, for stars built with the EOS from [16]. See [1] for references. 
is covering the inner part of the crust with some extension in the outermost layers of the core. The proton ${ }^{1} S_{0}$ gap is also certainly present in the outer core and may or may not reach the center of the star, depending on the specific pairing model considered and on the central density of the star. Figure 4 shows a representative sample of theoretical predictions for the associated critical temperature $T_{c}$. Neutron pairing in the ${ }^{3} P_{2}$ state is much more delicate and there is a very wide range of predictions as is illustrated by the examples shown in Figure 4. As shown by Baldo et al [14] the poor understanding of the nucleon-nucleon interaction in the ${ }^{3} P_{2}$ state in vacuum by itself results in a wide range of predictions for $T_{c}$, illustrated by the three curves labeled "a", "b", and "c" in Figure 4. Moreover, in medium effects were recently shown to have a dramatic effect on this gap [15] which may result to be vanishingly small.

The dramatic effect of pairing on the cooling comes from the gap it introduces in the single particle excitation spectrum which results in a strong suppression of both the specific heat and the neutrino emissivity of the paired component. When $T \ll T_{c}$ this suppression is similar to a Boltzmann factor $\exp (-\Delta / k T)$ and in general it is taken into account accurately by multiplying the relevant $c_{v}$ 's and $q_{\nu}$ 's by appropriate "control functions" (See Fig. 5).

\subsection{The Pair Breaking and Formation ("PBF") neutrino emission process}

The occurrence of pairing has a third effect, beside the suppression of $c_{v}$ and $q_{\nu}$, which is the emission of $\nu-\bar{\nu}$ pairs at temperature below, but close to, $T_{c}$ produced by the formation and breaking of Cooper pairs, the "PBF" process $[17,18]$. This process leads to a sudden increase of the neutrino emission in a given layer, when $T$ reaches $T_{c}$, which can largely dominates over the emission from the MUrca process. For example, in the case of the neutron ${ }^{3} P_{2}$ pairing its emissivity is

$$
q_{\nu}^{\mathrm{n}^{3} \mathrm{P}_{2}}=8.6 \times 10^{21}\left(\frac{\rho}{\rho_{0}}\right)^{1 / 3}\left(\frac{m_{n}^{*}}{m_{n}}\right) \times \tilde{F}_{3 \mathrm{P}_{2}}\left(T / T_{c}\right)\left(\frac{T}{10^{9} \mathrm{~K}}\right)^{7}
$$

The control functions $\tilde{F}$ are plotted in Fig. 5 and describe the onset of the process when $T$ reaches $T_{c}$ and its suppression when $T \ll T_{c}$. Comparing the emissivities given above with the MUrca process in Table 1 one sees that these PBF processes can dominate the neutrino emission and we will see in the next section that they are an essential ingredient of the Minimal Scenario. 

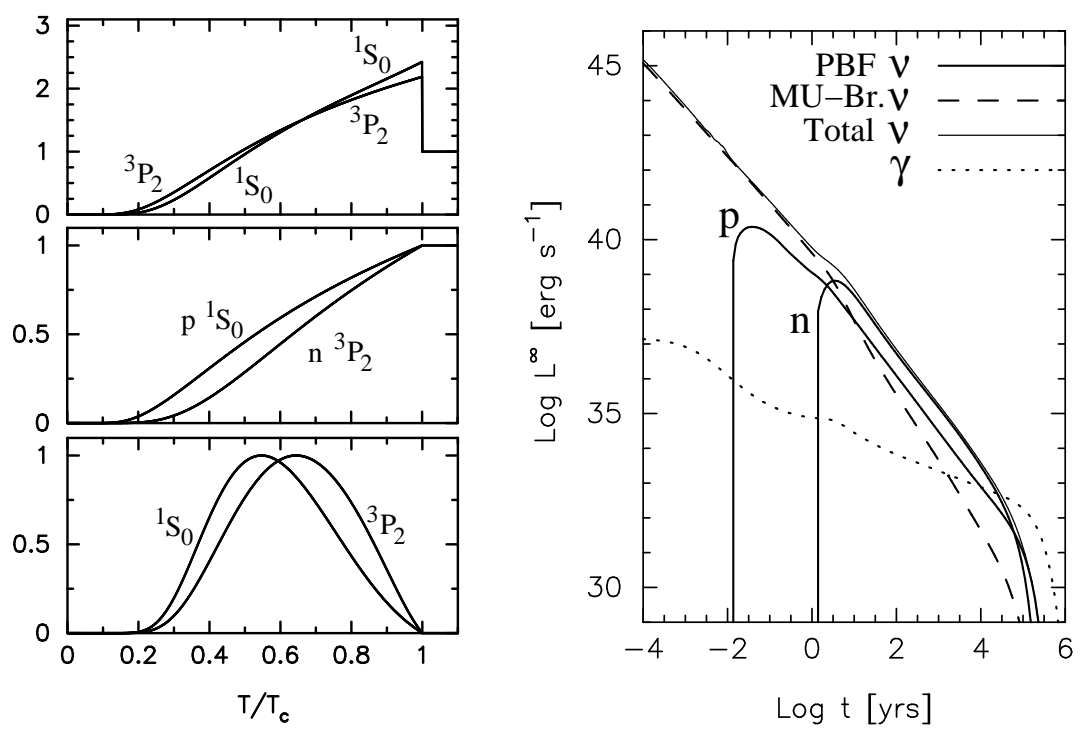

Figure 5. Left panel: control functions, for pairing in the ${ }^{1} S_{0}$ and ${ }^{3} P_{2}$ phases, of the specific heat (top), MUrca process (middle) and PBF process (bottom). Right panel: comparison of the neutrino luminosities from the two PBF processes due to neutron ${ }^{3} P_{2}$ and proton ${ }^{1} S_{0}$ gap (the neutron ${ }^{1} S_{0}$ gap contribution is small and not shown here), and from the MUrca processes, with the total neutrino luminosity and the photon luminosity (neutron ${ }^{3} P_{2}$ gap from model "a" and proton ${ }^{1} S_{0}$ pairing from model $\mathrm{AO}$, as labeled in Fig 4).

\subsection{The specific heat}

For normal (i.e., unpaired) degenerate spin $\frac{1}{2}$ fermions of type "i", the specific heat (per unit volume) is

$$
c_{i, v}=N_{i}(0) \frac{\pi^{2}}{3} k_{B}^{2} T=\frac{m_{i}^{*} n_{i}}{p_{i, F}^{2}} \pi^{2} k_{B}^{2} T
$$

Most of the specific heat of the star is provided by the core and, in absence of "exotic" matter, nucleons contribute about $90 \%$ of it while leptons ( $e$ and $\mu)$ share the remaining. Once neutrons and/or protons go into a paired state their specific heat is strongly altered: when $T$ reaches $T_{c}$ there is a discontinuity in $c_{v}$ which suddenly increases but when $T \ll T_{c}$ it becomes exponentially suppressed. This effect is also accurately taken into account by introducing a multiplicative "control function" plotted in Fig. 5. It is important to notice that even in case both neutrons and protons are paired in the whole core we still have the contribution of the leptons which remains untouched, i.e., pairing can reduce the total $C_{v}$ by at most $90 \%$ while $L_{\nu}$ 
can be suppressed by many orders of magnitude in case baryons involved in all the important processes are paired.

\section{The Minimal Scenario}

The physical ingredients presented in the previous section constitute all the essential input for the Minimal Scenario. Since, by definition, this scenario does not admit any enhanced neutrino emission the cooling history of a neutron star has only a very weak dependence on its mass. Moreover, the supranuclear EOS is also well constrained within this scenario so that we can generally simply study the evolution of a "canonical" $1.4 M_{\odot}$ neutron star. All results presented here are based on the EOS from APR [16]. What is not constrained by the requirement of the Minimal Scenario is:

\section{A) the chemical composition of the envelope and B) the pairing state of the nucleons}

and the large uncertainties in these two physical ingredients are, by far, the most important sources of uncertainty in the theoretical predictions of the Minimal Scenario.

The effect of the envelope is illustrated in the left panel of Fig. 6 for the two extreme cases of a star with an envelope consisting only of heavy elements (marked as "H") and with an envelope containing a maximum amount of light elements (marked as "L"). The important features to note are:

A1) first, at age inferior to $10^{5} \mathrm{yrs}$, both stars have the same central temperature but the "L" model has a higher $T_{e}$ : this correspond to the neutrino cooling era where $L_{\nu}$ drives the cooling, hence the same $T_{\text {center }}$ for both stars, and the surface temperature simply follows the interior evolutions, hence a higher $T_{e}$ in presence of a less insulating light element envelope, and

A2) later, during the photon cooling era when $L_{\gamma} \gg L_{\nu}$, the cooling trajectories get inverted since the light element envelope results in a much larger photon luminosity and hence faster cooling.

With an envelope containing a smaller amount of light elements we obtain an intermediate evolution and in case we allow for a time evolution of the amount of light elements the evolution can switch from one trajectory to the other.

The overall effect of pairing is illustrated in the three cooling curve plotted in the right panel of Fig. 6 . The two important features to note are: 
B1) comparing the model without pairing with the model with pairing but without the PBF process taken into account, one sees the effect of the suppression of the MUrca neutrino process resulting in a warmer star during the neutrino cooling era while during the photon cooling era the results are inverted because of the suppression of $C_{v}$ from the pairing, and B2) once the neutrino emission from the PBF process is taken into account the cooling is strongly enhanced during the neutrino cooling era, confirming the results of Fig 5 (right panel) that this PBF process can be much more intense than the MUrca one, and finally during the photon cooling era the two paired models, with and without PBF, join once they have forgotten their previous neutrino cooling history.

Of course the PBF process is always acting in presence of pairing and it has been artificially turned-off for this figure, but its efficiency depends on the actual size of the gap, i.e., the actual profile of $T_{c}$ for either neutrons or protons, and the gaps used in Fig 5 have been chosen to maximize the effect.
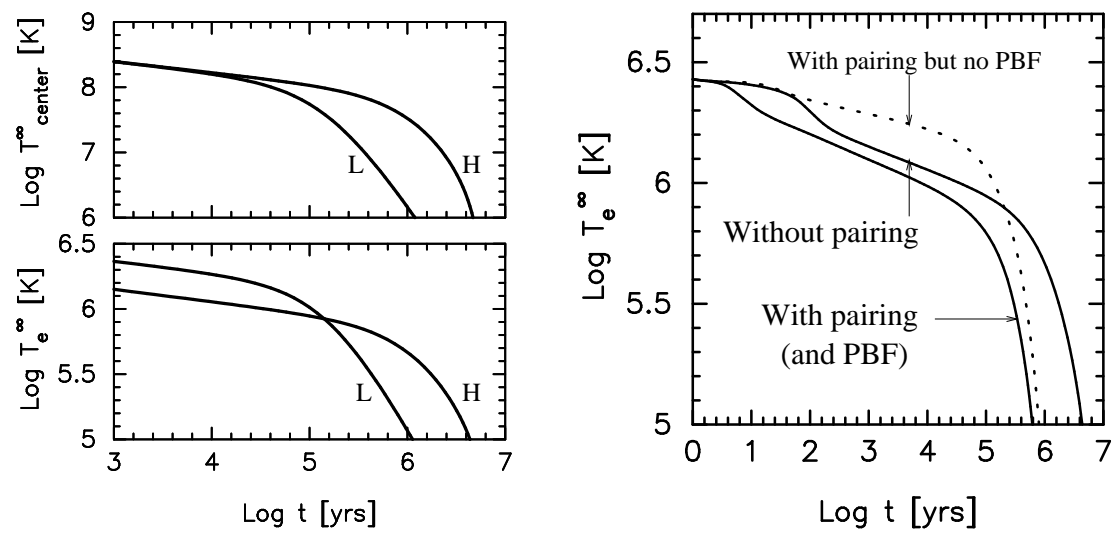

Figure 6. Left panel: effect of the envelope chemical composition in the cooling. Right panel: effect of nucleon pairing on the cooling. (See text for description.)

An extensive comparison of the predictions of the Minimal Scenario with the data presented in Sec. 2 is shown in Fig 7. For the reasons discussed in Sec. 2 results are plotted twice, as $T_{e}$ vs age (left panels) and $L$ vs age (right panels). The uncertainties due to the exact extent of nucleon pairing are better assessed by classifying the possible models into three families depending on the size of the neutron ${ }^{3} P_{2}$ gap since this is the most uncertain one: a vanishing gap and the schematic models "a" and "b" of 
Fig. 4. For each of these three families variations of the neutron and proton ${ }^{1} S_{0}$ gaps generate a set of closely packed curves and, given the uncertainty about the envelope chemical composition each set is shown twice, assuming an envelope made of heavy elements and an envelope with a maximum amount of light elements. (For clarity only these two extreme cases of envelope are shown, but any trajectory inbetween is possible.)

The overall agreement between theory and data is quite good, which I personally find in itself amazing considering that this is REAL THEORY: these calculations culminate several decades of works from hundreds of physicists and astrophysicists based essentially on only a handful of observational facts (the very existence of "neutron stars", several mass measurements and their extreme compactness known from pulsar timing, ...).

The three sets of models, for the three different neutron ${ }^{3} P_{2}$ gaps, are quite similar but do show some essential differences. When considering young stars, particularly J0205+6449 (in 3C58), PSR 0833-45 (in Vela) and PSR 1706-44, one sees that the models with the ${ }^{3} P_{2}$ gap "a" are very close to the upper limits of Vela and 3C58 while the difference is larger with the other two gaps. The interpretation of the data of PSR 1706-44 is more ambiguous due to the presently large uncertainty on both it temperature (or luminosity) and age. Since no thermal emission has been actually detected from $3 \mathrm{C} 58$ it is more prudent to consider it on a $L$-age plotted where the discrepancy with the theoretical predictions is actually the largest.

Several of the older objects may have temperatures higher than some of the theoretical predictions of the Minimal Scenario. This may be attributed to an erroneous age, considering that the only information we have bout their possible age is the spin-down time scale which can be very misleading. Another possibility is that some "heating mechanism' is at work which converts rotational, or magnetic, energy into heat.

\section{Conclusions}

In the Quest for New States of Dense Matter we have performed an extensive study of the thermal evolution of isolated neutron stars under the hypothesis that no new phase is present and tried to find some incompatibility of the results of this assumption with the best presently available data on cooling neutron stars. The final results, presented in Fig. 7 show now striking incompatibility with, nevertheless two objects, J0205+6449 (in 3C58) and PSR 0833-45, which are conspicuously lower than our predictions. Given the capability of both Chandra and XMM-Newton one can 

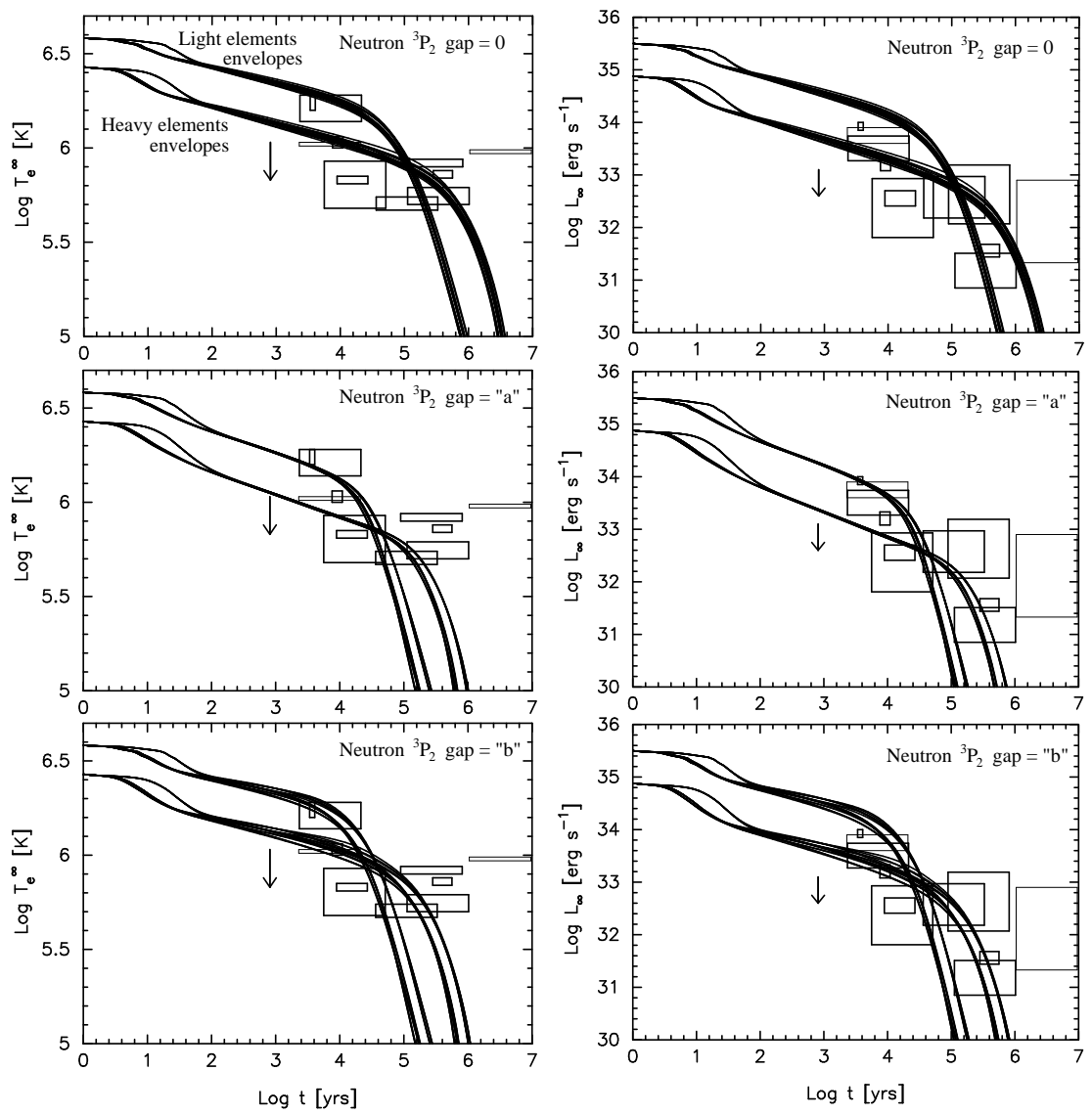

Figure 7. Comparison of predictions of the Minimal Scenario with data. Left panels: effective temperature at infinity $T_{e}^{\infty}$ vs. age. Right panels: luminosity at infinity $L_{\infty}$ vs age. The upper, middle, and lower panels correspond to three different assumption about the size of the neutron ${ }^{3} P_{2}$ gap as indicated in the panels. In each panel the two sets of curves correspond to the two extreme models of envelope chemical composition: light elements or heavy elements, as labeled in the upper left panel. For each set of curves, the different 15 curves correspond to different choices of the neutron (3 cases) and proton (5 cases) ${ }^{1} S_{0}$ gaps. $1.4 M_{\odot}$ star built with the EOS of APR.

have the hope that in the near future either more such objects will be found (see, e.g., Kaplan et al. [19]) or that more data on these two conspicuous stars will permit more detailed studies and confirm them as star(s) which encompass a new state of dense matter. 


\section{Acknowledgments}

Work partially supported by grants from UNAM-DGAPA (\#IN112502) and Conacyt (\#36632-E). The author also wants to thank the organizers of this Workshop for the invitation and financial support.

\section{References}

1. Page, D., Lattimer, J. M., Prakash, M., \& Steiner, A. W. To be published in Astrophys. J. Suppl. [astro-ph/0403657]

2. Van Kerkwijk, M. This volume

3. Page, D., Prakash, M., Lattimer, J. M.,\& Steiner, A. W. Phys. Rev. Lett. 85, $2048(2000)$

4. Page, D., \& Usov, V. V. Phys. Rev. Lett. 89, 131101 (2002)

5. J. Drake, This volume

6. Geppert, U., Kücker, M., \& Page, D. Submitted to Astronom. E Astrophys. [astro-ph/0403441]

7. Gudmundsson, E.H., Pethick, C.J. \& Epstein, R.I. Astrophys. J. 272, 286 (1983)

8. Potekhin, A. Y., Chabrier, G., \& Yakovlev, D. G. Astron. ES Astrophys. 323, 415 (1997)

9. Potekhin, A. Y., \& Yakovlev, D. G. Astron. \&3 Astrophys. 374, 213 (2001)

10. Page, D. Astrophys. J. 442, 273 (1995)

11. Lattimer, J. M., Pethick, C. J., Prakash, M., \& Haensel, P. Phys. Rev. Lett. 66, 2701 (1991)

12. Pethick, C. J. 1992, Rev. Mod. Phys., 64, 1133

13. Yakovlev, D. G., Kaminker, A. D., Gnedin, O. Y., \& Haensel, P. Phys. Rep. 354, 1 (2001)

14. Baldo, M., Elgarøy, Ø., Engvik, L., Hjorth-Jensen, M., \& Schulze, H.-J. Phys. Rev. C58, 1921 (1998)

15. Schwenk, A., \& Friman, B. Phys. Rev. Lett. 92, 082501 (2004)

16. Akmal, A., Pandharipande, V. R., \& Ravenhall, D. G. Phys. Rev. C58, 1804 (1998)

17. Flowers, E., Ruderman, M., \& Sutherland, P. Astrophys. J. 205, 541 (1976)

18. Voskresensky, D. N., \& Senatorov, A. V. Sov. J. Nucl. Phys. 45, 411 (1987)

19. Kaplan, D. L., et al. This volume and [astro-ph/0403313] 